\title{
Neuro-otological and psychiatric abnormalities in a community sample of people with dizziness: a blind, controlled investigation
}

\author{
L Yardley, J Burgneay, I Nazareth, L Luxon
}

Department of Psychology, University College London, Gower St, London, UK L Yardley

Hearing and Balance Centre, Institute of Sound and Vibration Research, University of Southampton Highfield, Southampton, UK

J Burgneay

Department of Primary Care and Population Sciences, Royal Free Hospital School of Medicine and University College London Medical School, Royal Free Hospital School of Medicine, Rowland Hill St, London, UK I Nazareth

Institute of Laryngology and Otology, University College London, 330/332 Gray's Inn Road, London, UK L Luxon

Correspondence to: Dr L Yardley, Department of Psychology, University College London, Gower St, London WC1E 6BT, UK.

Received 27 November 1998 and in revised form 12 March 1998

Accepted16 April 1998

\begin{abstract}
Objectives-To determine neurootological and psychiatric abnormalities associated with complaints of dizziness in an epidemiological community sample of people of working age, and the extent of comorbidity between neuro-otological and psychiatric dysfunction.

Method-A survey of 3884 people randomly selected from six general practice lists identified 262 people with significant dizziness, from which a subsample of 15 men and 22 women were recruited for testing. Dizzy subjects were evaluated by blind neuro-otological testing, computerised dynamic posturography, a computerised psychiatric assessment, neuro-otological and general medical examination, and diagnosis. An age matched control group of 18 men and 22 women underwent the same evaluation.

Results-Tests of auditory, vestibular, and oculo-motor function did not discriminate between dizzy subjects and controls, but dizzy subjects had significantly worse balance on posturographic testing, more diagnoses of medical disorder, and a higher prevalence of psychiatric morbidity.

Conclusions-The findings suggest that dizziness in the community is typically characterised by mild physical disorder accompanied by some psychiatric disturbance. As the combination of minor physical and psychiatric disorder is known to be unusually persistent and handicapping, treatment programmes must be provided for this prevalent syndrome, perhaps by a partnership between primary care and neuro-otological and psychiatric hospital outpatient clinics with experience and expertise in the diagnosis and management of dizziness and psychiatric disturbance.

(F Neurol Neurosurg Psychiatry 1998;65:679-684)
\end{abstract}

Keywords: vestibular; psychiatric disorder; dizziness; anxiety; panic; balance

Dizziness is a very common symptom, reported by up to one in three elderly people,${ }^{1-3}$ and over one in five people of working age. ${ }^{45}$ However, $40 \%$ of people in the community with handicapping dizziness fail to consult their family doctor, ${ }^{4}$ and only a few of those who do so are referred for specialist testing. ${ }^{6}$ Consequently, the prevalence of neuro-otological abnormalities in the general population re- mains unknown, and the prevalence of undiagnosed potentially treatable neuro-otological disorders in the community has not been determined.

Most studies of the distribution of test abnormalities and diagnoses among dizzy patients have been based on people attending hospital outpatient clinics, ${ }^{7-13}$ who are clearly not typical of dizziness in the wider community. Whereas cases of dizziness seen in primary care are more representative of the general population, it is often difficult for primary care physicians to provide a definitive diagnosis. In a United States study, the principal diagnoses provided for mixed age dizzy patients in primary care were labyrinthitis, cardiovascular disorder (especially hypertension), and dizziness of unknown origin. ${ }^{14}$ Less than a third of patients with dizziness in a United Kingdom primary care study had specific neurootological diagnoses, most being classified as vertigo or dizziness of unknown origin. ${ }^{15} \mathrm{How}-$ ever, a unique in depth otological and psychiatric investigation of 75 unselected patients complaining of dizziness in primary care disclosed that over half had evidence of vestibular dysfunction. ${ }^{16}$

Recent hospital based research suggests that there is considerable comorbidity of physical and psychiatric disorder in patients with dizziness. The prevalence of psychiatric disorders (particularly anxiety, panic disorder, and agoraphobia) among patients with balance disorders is much higher than in the general population. ${ }^{70-1217}$ The prevalence of dysfunction of the balance system among patients with panic disorder and agoraphobia is similarly high. ${ }^{18-22}$ Indeed, attempts to distinguish between organic and "psychogenic" dizziness can prove unproductive, as these patients often present with a chronic syndrome of combined physical and psychiatric dysfunction resulting from various psychophysiological and somatopsychic processes, which may be mediated in part by central neuroanatomical links between the vestibular and autonomic nervous systems. ${ }^{23-26}$ However, the high levels of psychiatric morbidity seen in hospital samples may be specific to this more severe group of patients, and the prevalence of this syndrome in primary care and the community is currently unknown.

The primary aim of the present study was to determine the nature and extent of neurootological and psychiatric abnormalities associated with dizziness in a sample of working age adults drawn from an epidemiological community sample. This sampling strategy would 
Table 1 Comparison of participants and non-participants on survey measures

\begin{tabular}{lll}
\hline & Participants & Non-participants \\
\hline Age (mean (SD)) & $39.43(11.8)$ & $38.41(11.7)$ \\
Sex (n (\%)): & $15(40.5)$ & $65(28.9)$ \\
$\quad$ Male & $22(59.5)$ & $160(71.1)$ \\
$\quad$ Female & $22(75.9)$ & $92(68.1)$ \\
Occupation (n (\%)): & $7(24.1)$ & $43(31.9)$ \\
$\quad$ Classes I-IIIN & & \\
Classes IIIM-V & $12(32.4)$ & $86(38.7)$ \\
Reported dizziness symptoms during the past & $24(64.9)$ & $157(70.7)$ \\
month (n (\%)): & $13(35.1)$ & $95(42.8)$ \\
$\quad$ Vertigo & $20(54.1)$ & $136(61.3)$ \\
Giddiness, lightheadedness & & \\
$\quad$ Syncope, fainting & $5(15.6)$ & $34(16.8)$ \\
$\quad$ Unsteadiness, falling & $11(34.4)$ & $56(27.7)$ \\
Dizziness duration (n (\%)): & $10(31.3)$ & $45(22.3)$ \\
$\quad$ Less than 6 months & $6(18.8)$ & $67(33.2)$ \\
$\quad$ Six months to 2 years & $1.4(2.59)$ & $1.8(3.35)$ \\
$\quad$ Two to 5 years & & \\
$\quad$ More than 5 years & &
\end{tabular}

enable us to determine to what extent our subsample was representative of dizzy people in the wider community, and in what ways (if any) it might deviate from the general population. The second objective of this study was to establish the extent of comorbidity of physical and psychiatric dysfunction, and to ascertain whether neuro-otological findings differed in those with psychiatric symptoms and those without. To obtain a community sample for detailed medical investigation the representativeness of which could be accurately assessed, we tested subjects identified in a survey of the prevalence and presentation of dizziness in a large random community sample. ${ }^{4}$ This allowed us to compare the demographic characteristics and symptomatology of those who were tested with the much larger community sample from which they were drawn. These dizzy subjects, and a control group of subjects roughly matched for age and sex, were given a comprehensive blind neuro-otological evaluation, plus an assessment of general medical condition and psychiatric status.

\section{Methods}

SUBJECTS

Dizzy subjects were recruited by means of two community surveys, in which screening questionnaires assessing symptoms of dizziness and handicap levels were sent to a random sample of one in three patients aged 18-64 listed in six general practices in socioeconomically diverse areas of north London. ${ }^{4}$ Respondents were considered eligible for testing if they reported dizziness and a handicap level greater than one (moderate to severe effects on at least one lifestyle dimension, or mild effects on at least two dimensions).

Of the 3884 people who participated in the screening surveys (representing a $70 \%$ response rate), 262 met the eligibility criteria for testing, and 71 agreed to come for testing. Of these, 17 did not attend, and 17 were excluded because they were unable to cease taking medication with psychoactive effects which might affect the test results. The final sample size was 37 , comprising 15 men and 22 women with an average age of 39.43 (SD 11.8) years. There was no significant difference between dizzy survey respondents who were tested and those who did not come for testing (table 1) on any survey variable except handicap levels, which were somewhat greater among those who were not tested $\left(t_{260}=2.47, \mathrm{p}=0.01\right)$.

The control subjects were 18 men and 22 women with an average age of 37.53 (SD 11.4) years, recruited by means of advertisement. Control subjects were screened to ensure that they had no visual or CNS impairment, had never had a diagnosis of vestibular dysfunction, and had experienced no dizziness during the past year.

The study was approved by the UCL/UCH committee on the ethics of human research, and all subjects gave written informed consent before participating. Subjects were instructed to refrain from taking alcohol or medication for 24 hours before testing.

\section{EQUIPMENT}

A Graystad GSI10 audiometer with TDH50P headphones was used for audiometry, and a Graystad GSI33 was employed for tympanometry. DC recordings of eye movements were obtained using $10 \mathrm{~mm} \mathrm{Ag} / \mathrm{AgCl}$ electrodes and a Gould Windograf recorder. Saccades and pursuit were tested using computer controlled light emitting diode stimuli in darkness. For optokinetic testing the subject was seated at the centre of a striped rotating drum (diameter 152 $\mathrm{cm}$, stripe width $10 \mathrm{~cm}$ ). For rotation testing, the subject was seated in a servo controlled rotating chair with DC motor, and belt drive for speed reduction. Caloric irrigation was performed using a Hortman Aquamatic water caloric stimulator, and postural stability was assessed by computerised dynamic posturography using the EquiTest ${ }^{\mathrm{TM}}$ system. ${ }^{27}$ Computerised psychiatric assessment was carried out using the PROgrammable Questionnaire SYstem (PROQSY) ${ }^{28}$ administered on a personal computer.

\section{AUDIO-VESTIBULAR TESTS}

Testing was carried out by an experienced audiological scientist (JB) who was blind to the subject's status (dizzy or control).

Pure tone audiometry, masked as necessary, was performed using the techniques recommended by the British Society of Audiology. Hearing loss was defined as hearing threshold worse than age related norm $\mathrm{s}^{29}$ by at least $5 \mathrm{~dB}$ at three frequencies or $10 \mathrm{~dB}$ at two frequencies, and was classified as "noise induced" if there was a peak hearing loss at $4-6 \mathrm{kHz}$ and a compatible history, and as "conductive" or "mixed" if there was an air-bone gap of at least $10 \mathrm{~dB}$ at three frequencies.

Middle ear pressure and tympanic membrane compliance were measured by immittance audiometry. The normal range for middle ear pressure was taken as between -100 and $+100 \mathrm{daPa}$, and normal compliance was defined as $0.3 \mathrm{ml}$ to $1.5 \mathrm{ml}$ of equivalent air volume.

Measurement of spontaneous nystagmus by electronystagmography (ENG) was conducted with eyes open in the light and the dark, and with eyes centre, and with $30^{\circ}$ to right and left. The average maximum slow phase velocity was 
Table 2 Dizzy subjects and controls with abnormal auditory test results

\begin{tabular}{lll}
\hline & $\begin{array}{l}\text { Dizzy } \\
\text { subjects } \\
(n(\%))\end{array}$ & $\begin{array}{l}\text { Control } \\
\text { subjects } \\
(n(\%))\end{array}$ \\
\hline Audiometry (hearing loss): & $6(17.1)$ & $3(7.7)$ \\
$\quad$ Bilateral sensorineural & $3(8.6)$ & $3(7.7)$ \\
Unilateral sensorineural & $2(5.7)$ & $2(5.1)$ \\
$\quad$ Noise induced & $2(5.7)$ & $7(17.9)$ \\
$\quad$ Conductive or mixed & $1(2.9)$ & $3(7.7)$ \\
Tympanometry: & $4(11.4)$ & $6(15.4)$ \\
$\quad$ Hypomobile eardrum & $2(5.7)$ & $2(5.1)$ \\
Hypermobile eardrum & & \\
Low middle ear pressure &
\end{tabular}

manually determined from the three beats of nystagmus with the highest velocity.

Optokinetic responses were assessed by gain (calculated from average maximum slow phase velocity) of the nystagmus induced by rotation of a striped drum around the subject for 15 seconds at $40 \%$ s, clockwise and anticlockwise. Response to sinusoidal rotation testing was assessed by gain of nystagmus induced by sinusoidal rotation in the dark, oscillation frequency $0.05 \mathrm{~Hz}$, maximum velocity $40 \%$ s. After six cycles in the dark, testing was repeated with a fixation light to evaluate vestibulo-ocular reflex (VOR) suppression. Response to impulsive rotation testing was measured by the time constant of the nystagmus induced by a sudden stop after constant velocity rotation of the subject at $60 \%$ s, clockwise and anticlockwise.

Caloric responses were assessed by duration of nystagmus induced by irrigation of the left and right ear canals for 40 seconds with warm $\left(44^{\circ} \mathrm{C}\right)$ and cold $\left(30^{\circ} \mathrm{C}\right)$ water, with mental alerting. Duration was determined by observation, firstly with fixation, and then with eyes open under Frenzel glasses.

Asymmetry on the rotation, optokinetic, and caloric tests was calculated using the Jongkees formula. ${ }^{30} \mathrm{~A}$ conservative criterion of response asymmetry of $10 \%$ or more was employed to classify responses as abnormal.

COMPUTERISED DYNAMIC POSTUROGRAPHY

Postural stability was evaluated by the maximum amplitude of sway (in the anteriorposterior direction) obtained during three trials under each of six successive perceptual conditions (the sensory organisation test ${ }^{27}$ ). These comprised ( $a$ ) eyes open, $(b)$ eyes closed, and $(c)$ vision "sway-referenced", all on a stable support surface, followed by the same three conditions with the support surface swayreferenced. The system's software averages sway over the 18 trials to yield a composite score expressed as a percentage, with $100 \%$ representing perfect stability. Sway was also compared across the different test conditions, using the standardised software routines to identify deficits in the use of visual, vestibular, or somatosensory information, and inability to ignore misleading visual information (visual preference). ${ }^{27}$

MEDICAL EXAMINATION AND DIAGNOSIS

Medical examination was carried out by an experienced clinician (LL) who was blind to the subject's history and status. Examination comprised assessment of: spontaneous nystagmus with and without fixation, pursuit, horizontal and vertical saccades, horizontal and vertical optokinetic nystagmus (elicited by a hand held drum), the Romberg test of postural stability, eyes open and eyes closed, the Hallpike test for benign paroxysmal positional nystagmus, otoscopy, the Rinne and Weber tests of hearing, the visual convergence and cover tests, cranial nerve tests, tests of freedom of cervical movement, pulse rate and rhythm, and blood pressure (defined as abnormal if systolic blood pressure $>160 \mathrm{~mm} \mathrm{Hg}$, or diastolic $>90 \mathrm{~mm} \mathrm{Hg}$ )

After testing was complete, the clinician took a full history and made a diagnosis on the basis of history, examination, and test results.

\section{COMPUTERISED PSYCHIATRIC ASSESSMENT}

An assessment of psychiatric status was carried out using PROQSY, a well validated computerised version of the clinical interview schedule, used to measure neurotic symptoms in community and general medical subjects. ${ }^{28}{ }^{31}$ Subjects are defined as cases if they obtain a total weighted score greater than 12, whereas specific neuroses are identified from scores $>2$ on the subsections of the interview.

Table 3 Gain (sinusoidal and optokinetic testing) and duration in seconds (impulsive and caloric testing) of nystagmus induced by rotary, optokinetic, and caloric stimulation, in dizzy subjects and controls

\begin{tabular}{|c|c|c|c|c|c|c|}
\hline \multirow[b]{2}{*}{ Test } & \multicolumn{3}{|c|}{ Control subjects } & \multicolumn{3}{|c|}{ Dizzy subjects } \\
\hline & Mean & $S D$ & $10 \%-90 \%$ & Mean & $S D$ & $10 \%-90 \%$ \\
\hline \multicolumn{7}{|l|}{ Optokinetic testing: } \\
\hline Drum moving anticlockwise & 0.889 & 0.186 & $0.63-1.18$ & 0.845 & 0.302 & $0.56-1.41$ \\
\hline Drum moving clockwise & 0.872 & 0.197 & $0.57-1.16$ & 0.853 & 0.286 & $0.45-1.18$ \\
\hline \multicolumn{7}{|l|}{ Sinusoidal rotation testing: } \\
\hline Chair moving anticlockwise & 0.643 & 0.257 & $0.24-0.97$ & 0.771 & 0.262 & $0.43-1.18$ \\
\hline Chair moving clockwise & 0.639 & 0.234 & $0.34-0.88$ & 0.692 & 0.236 & $0.35-0.96$ \\
\hline \multicolumn{7}{|l|}{ Impulsive rotation testing: } \\
\hline Following anticlockwise movement & 16.73 & 7.88 & $5.8-24.8$ & 19.21 & 7.45 & $10.5-25.5$ \\
\hline Following clockwise movement & 17.50 & 4.63 & $11.7-23.5$ & 20.92 & 6.90 & $12.0-31.5$ \\
\hline \multicolumn{7}{|l|}{ Caloric testing: } \\
\hline Hot irrigation, left ear (EO) & 119.60 & 29.79 & $93-157$ & 120.73 & 21.37 & 91-149 \\
\hline Hot irrigation, right ear (EO) & 116.11 & 33.83 & $90-152$ & 125.10 & 23.50 & $100-170$ \\
\hline Cold irrigation, left ear (EO) & 95.60 & 42.45 & $0-134$ & 108.45 & 21.59 & $80-140$ \\
\hline Cold irrigation, right ear (EO) & 96.76 & 44.55 & $0-136$ & 109.82 & 27.74 & 89-132 \\
\hline Hot irrigation, left ear (EC) & 141.53 & 33.29 & $107-176$ & 142.00 & 27.56 & $105-180$ \\
\hline Hot irrigation, right ear (EC) & 144.16 & 35.69 & $107-190$ & 147.83 & 26.73 & $120-200$ \\
\hline Cold irrigation, left ear (EC) & 136.79 & 34.17 & $107-178$ & 141.66 & 28.54 & $110-180$ \\
\hline Cold irrigation, right ear (EC) & 161.64 & 30.73 & $126-200$ & 144.48 & 37.11 & $120-185$ \\
\hline
\end{tabular}


Table 4 Asymmetry of responses to vestibulo-ocular tests in dizzy subjects and controls

\begin{tabular}{|c|c|c|c|c|c|c|}
\hline \multirow[b]{2}{*}{ Test } & \multicolumn{3}{|c|}{ Control subjects } & \multicolumn{3}{|c|}{ Dizzy subjects } \\
\hline & Median & $\begin{array}{l}\text { Interquartile } \\
\text { range }\end{array}$ & $\begin{array}{l}\text { Abnormal } \\
\text { asymmetry } \\
\geqslant 10 \%(n(\%))\end{array}$ & Median & $\begin{array}{l}\text { Interquartile } \\
\text { range }\end{array}$ & $\begin{array}{l}\text { Abnormal } \\
\text { asymmetry } \\
\geqslant 10 \%(n(\%))\end{array}$ \\
\hline \multicolumn{7}{|l|}{ Rotation testing: } \\
\hline Optokinetic & 0.100 & $0.04-0.15$ & $2(7.4)$ & 0.100 & $0.05-0.18$ & 0 \\
\hline Sinusoidal & 0.150 & $0.05-0.26$ & $6(21.4)$ & 0.125 & $0.05-0.26$ & $7(19.4)$ \\
\hline Impulsive & 3.5 & $1-9$ & $8(34.8)$ & 5 & $2-12$ & $4(16.7)$ \\
\hline \multicolumn{7}{|l|}{ Caloric testing: } \\
\hline Canal paresis (EO) & 3.1 & $1.0-6.4$ & $5(17.9)$ & 2.5 & $0.8-6.4$ & $3(10.0)$ \\
\hline Canal paresis (EC) & 4.6 & $1.0-7.8$ & $6(21.4)$ & 5.6 & $1.9-7.8$ & $4(15.6)$ \\
\hline $\mathrm{DP}(\mathrm{EO})$ & 2.5 & $1.2-4.8$ & $3(10.7)$ & 2.2 & $1.2-4.8$ & $4(13.3)$ \\
\hline $\mathrm{DP}(\mathrm{EC})$ & 4.2 & $2.6-8.2$ & $2(7.1)$ & 3.7 & $2.6-8.2$ & $4(12.5)$ \\
\hline
\end{tabular}

Asymmetry on rotation tests was calculated by averaging difference in gain of responses to left and right. Asymmetry on the caloric test is represented by conventional calculations of percentage canal paresis and directional preponderance (DP).

\section{STATISTICAL ANALYSIS}

The $\chi^{2}$ test was used to compare frequency of abnormal test results in dizzy and control subjects, the non-parametric Mann-Whitney $U$ test to compare number of vestibular test abnormalities in each group. Dizzy subjects and controls were compared on six global measures, and so to minimise type I errors a significance level of $0.008(0.05 / 6)$ was adopted, consistent with the Bonferroni procedure. Because some test subjects did not complete all the tests, actual numbers of subjects compared on each individual test are reported below, and percentages given are proportions of those tested.

\section{Results}

There was no significant difference in the proportion of dizzy subjects (16/37) and controls (20/40) who had some auditory abnormality $\left(\chi_{1}^{2}=0.35, p=0.55\right)$; table 2 gives details of the abnormalities found.

On ENG testing, four dizzy subjects and four controls had some minor, inconsistent nystagmus in the absence of fixation. Parameters of the eye movements induced by rotation, optokinetic, and caloric testing are shown in table 3, which shows that both mean values and variability were similar among dizzy and control subjects. Table 4 gives nonparametric indices of the distribution of asymmetry on the vestibulo-ocular tests, which was also similar in both groups. The number of vestibular test abnormalities/subject was not significantly different in dizzy and control subjects $(U=688.0, \mathrm{p}=0.57) ; 11$ dizzy subjects and 10 controls had one abnormal finding, five dizzy subjects and seven controls had two abnormal findings, and four dizzy subjects and two controls had three or four abnormal findings.

The proportion of dizzy subjects with abnormal composite posturography scores was significantly greater than in the controls

Table 5 Dizzy subjects and controls with abnormal scores on computerised dynamic posturography

\begin{tabular}{lll}
\hline & $\begin{array}{l}\text { Dizzy subjects } \\
(n=35)(n(\%))\end{array}$ & $\begin{array}{l}\text { Control subjects } \\
(n=40)(n(\%))\end{array}$ \\
\hline $\begin{array}{l}\text { Composite score } \\
\text { Vestibular deficit pattern }\end{array}$ & $19(54.3)$ & $8(20.0)$ \\
$\begin{array}{l}\text { Visual deficit pattern } \\
\begin{array}{l}\text { Somatosensory deficit } \\
\text { pattern }\end{array}\end{array}$ & $7(18.9)$ & $8(20.0)$ \\
Visual preference & $4(11.4)$ & 0 \\
\hline
\end{tabular}

$\left(\chi_{1}^{2}=9.52, p=0.002\right)$, and dizzy subjects tended to have patterns of sway indicating deficient use of vestibular, visual, and somatosensory information (table 5). Only two dizzy subjects, and no controls, had "aphysiological" patterns of sway, as indicated by abnormal sway on the easy test conditions (eyes open and eyes closed with no platform or visual surround movement).

Although at least one abnormality was found on examination in $10(29.4 \%)$ of the 34 dizzy subjects who had a complete examination and only four $(11.1 \%)$ of the 36 control subjects who had a complete examination, this difference did not reach significance $\left(\chi_{1}^{2}=3.66\right.$, $\mathrm{p}=0.056)$. Nevertheless, the distribution of abnormal findings (table 6) showed a trend towards greater prevalence of impaired cervical movement and abnormalities on tests of balance system function among dizzy subjects.

There was a highly significant difference in the diagnostic classification of dizzy subjects and controls $\left(\chi_{1}^{2}=25.3, p<0.0001\right)$, with $83.9 \%$ of patients receiving diagnoses of some kind of dysfunction, compared with just $22.2 \%$ of controls (table 7). There were few clear cut cases of neuro-otological disorders; only six dizzy subjects gave a classic history of true vertigo, and the remaining vestibular diagnoses were based on abnormal posturography and vestibular test results or a history suggestive of impaired compensation after minor vestibular dysfunction. The classification of "other medical conditions"

Table 6 Prevalence of abnormalities on medical examination in control and dizzy subjects

\begin{tabular}{lll}
\hline & $\begin{array}{l}\text { Dizzy } \\
\text { subjects }\end{array}$ & $\begin{array}{l}\text { Control } \\
\text { subjects }\end{array}$ \\
\hline Balance system tests: & & \\
$\quad$ Spontaneous nystagmus & 0 & 0 \\
Optokinetic nystagmus & 0 & 0 \\
Pursuit eye movements & 2 & 0 \\
Saccadic eye movements & 1 & 0 \\
Hallpike test & 0 & 0 \\
Romberg test & 2 & 0 \\
Otological tests: & 2 & 0 \\
Otoscopy & 0 & 0 \\
Rinne & 4 & 3 \\
Weber & & \\
Visual system tests: & 0 & 0 \\
Convergence & 1 & 2 \\
Cover & 0 & \\
General medical tests: & & 0 \\
Cranial nerves & 5 & 0 \\
Cervical movements & 0 & 1 \\
Pulse rate & 1 & 0 \\
Pulse rhythm & 1 & 1 \\
Blood pressure & & \\
\hline
\end{tabular}


Table 7 Diagnostic classifications in dizzy subjects with and without psychiatric abnormalities, and controls

\begin{tabular}{llll}
\hline Primary diagnosis & $\begin{array}{l}\text { Dizzy subjects } \\
\text { (no psychiatric } \\
\text { abnormalities; } n=18)\end{array}$ & $\begin{array}{l}\text { Dizzy subjects } \\
\text { (abnormal psychiatric } \\
\text { test scores; } n=13)\end{array}$ & $\begin{array}{l}\text { Control subjects } \\
(n=36)\end{array}$ \\
\hline $\begin{array}{l}\text { No dysfunction } \\
\begin{array}{l}\text { Peripheral vestibular } \\
\text { dysfunction }\end{array}\end{array}$ & 4 & 1 & 24 \\
$\begin{array}{l}\text { Central dysfunction } \\
\text { Psychiatric dysfunction }\end{array}$ & 8 & 7 & 8 \\
Other medical conditions & 1 & 2 & 0 \\
& 4 & 1 & 0 \\
\hline
\end{tabular}

Table 8 Abnormal computerised psychiatric assessment scores in dizzy subjects and controls

\begin{tabular}{lll}
\hline & $\begin{array}{l}\text { Dizzy subjects } \\
(n(\%))\end{array}$ & $\begin{array}{l}\text { Control subjects } \\
(n(\%))\end{array}$ \\
\hline Somatic symptoms & $21(60.0)$ & $4(11.1)$ \\
Worry about physical health & $14(40.0)$ & $5(13.9)$ \\
Fatigue & $30(85.7)$ & $12(33.3)$ \\
Irritability & $26(74.3)$ & $10(27.8)$ \\
Poor concentration & $21(60.0)$ & $10(27.8)$ \\
Depression & $16(45.7)$ & $5(13.9)$ \\
Phobias & $13(37.1)$ & $1(2.8)$ \\
Worry & $22(62.9)$ & $17(47.2)$ \\
Anxiety & $17(48.6)$ & $8(22.2)$ \\
Compulsions & $3(8.6)$ & $2(5.6)$ \\
Obsessions & $9(25.7)$ & $3(8.3)$ \\
\hline
\end{tabular}

comprised cases of head injury, vascular disease, suspected anaemia, hormonal change, and arthritis. The diagnoses of vestibular dysfunction in eight control subjects were based mainly on abnormalities on vestibular testing, although two had a history of second degree head injury and one had had an acoustic neuroma removed.

More than three times as many dizzy patients ( 16 out of the 35 tested) as controls (five out of the 36 tested) had abnormal psychiatric test scores $\left(\chi_{1}^{2}=8.62, \mathrm{p}=0.003\right)$. The distribution of abnormalities is shown in table 8 ; there was a much higher prevalence of dizzy patients in every category apart from "worry" and "compulsions". The most common problems among dizzy subjects were somatic symptoms, worry, fatigue, and poor concentration. The greatest difference in prevalence in the two groups was in phobias, as only one control subject but 13 dizzy subjects reported phobic behaviour.

Finally, to determine whether the dizzy subjects with psychiatric dysfunction differed from those without, the dizzy subjects were split into those with an abnormal total score on the computerised psychiatric assessment $(n=16)$ and those without $(n=19)$. No differences between these two groups were found on any of the global neuro-otological assessments, or in diagnoses (table 7).

\section{Discussion}

The variables which best discriminated between the dizzy subjects and the controls were computerised dynamic posturography, computerised psychiatric assessment, and the diagnosis. Very few patients had purely psychogenic symptoms ${ }^{32}$; most had deficient postural control or signs of some balance system dysfunction or medical disorder which could account for their dizziness. However, gross vestibular abnormalities were rare in this sample, whereas neurotic disturbance was extremely common. Moreover, patients could not be readily classi- fied as having dizziness due either to physical or psychiatric dysfunction, as signs of balance system dysfunction were as common among those with psychiatric disturbance as among those without.

The clinical profile these results suggest is of prevalent, mild combined physical and psychiatric disorder. This mixed aetiology is remarkably similar to that found in samples of dizzy patients drawn from hospital outpatient clinics ${ }^{912}$ and primary care. ${ }^{16}{ }^{26}$ Indeed, there is accumulating evidence that the extent to which mild dizziness becomes a chronic clinical problem depends mainly on the patient's psychological reactions to symptoms, ${ }^{433}$ as there is typically a high prevalence of minor vestibular abnormalities among control subjects who deny any history of significant dizziness. ${ }^{19} 21$ Transient, weak vestibular symptoms may hence be a common experience, but become a contributory factor for psychiatric disturbance, complaints of illness, and persisting handicap only in those who are predisposed to react adversely to disorientation, whether because of personality traits, behavioural responses, subclinical deficits in perceptual-motor capabilities or cognitive processing, or excessive autonomic nervous system reactivity. ${ }^{70} 23-25$

The syndrome of mixed physical and psychiatric disturbance disclosed by this study bears some similarity to the proposed diagnostic category of "phobic postural vertigo". ${ }^{34}$ However, phobic postural vertigo is described as psychogenic and associated primarily with an obsessive-compulsive personality, whereas most of our dizzy subjects had some evidence of organic dysfunction, and obsessivecompulsive tendencies were not prominent in our sample. We found various forms of psychiatric disturbance, and in particular a much higher prevalence of phobia than in the control group. Several previous studies have documented an association between objective balance system dysfunction and the development of "space and motion phobia" or "situational agoraphobia", which may result from the anxiety felt by those with deficient balance or orientation capabilities when exposed to disorienting environments such as motorways or shopping malls. ${ }^{16-21}$ In addition, a very high proportion of dizzy subjects complained of fatigue and difficulty in concentrating. This is an intriguing finding, in the light of recent experimental evidence showing that orientation and postural control make significant demands on attention and central processing capacity. ${ }^{35}{ }^{36}$ Symptoms suggesting mental exhaustion or overload may therefore reflect the need for dizzy patients to expend extra mental effort on the perception of orientation and control of eye and body movement.

In conclusion, it is apparent that whereas the prevalence of gross balance system abnormality is lower among dizzy people in the community than among patients referred to specialist clinics, the combination of mild physical disorder and psychiatric dysfunction is widespread. Indeed, the prevalence of physical and psychiatric disorder in this population may have been slightly underestimated by our 
study, as handicap levels were somewhat lower in the subsample tested than among dizzy people identified by the survey who did not come for testing. Despite the absence of gross physical or psychiatric pathology, this syndrome constitutes a far from trivial health problem, as the mixture of psychiatric and balance disorder is more persistent and handicapping than either complaint in isolation. ${ }^{4911}$ There is consequently a need to develop programmes of combined physical and psychological therapy which can more precisely meet the needs of this large, and at present largely untreated, ${ }^{4}$ patient population. Further research is needed to determine how such treatment can best be delivered, perhaps by a partnership between primary care and neuro-otological and psychiatric hospital outpatient clinics with experience and expertise in the diagnosis and management of dizziness and psychiatric disturbance.

We thank Drs C O'Mahoney, E Raglan, Y Silove, K Rajput, and A Thambapillai for assisting with patient assessment, and the Sir Health Authority for their financial support for this study.

1 Grimby A, Rosenhall U. Health-related quality of life and dizziness in old age. Gerontology 1995;41:286-98.

2 Sloane P, Blazer D, George LK Dizziness in a community elderly population. f Am Geriatr Soc 1989;37:101-8.

3 Weindruch R, Korper SP, Hadley E. The prevalence of dysWeindruch R, Korper SP, Hadley E. The prevalence of dys-
equilibrium and related disorders in older persons. Ear Nose Throat f 1989;68:925-9.

4 Yardley L, Owen N, Nazareth I, et al. Prevalence and presentation of dizziness in a general practice community sample of working age. Br F Gen Pract 1998;48:1131-5.

5 Patrick DL, Peach H. Disablement in the community. Oxford: Oxford University Press, 1989

6 Sloane PD, Dallara J, Roach C, et al. Management of dizziness in primary care. 7 Am Board Fam Pract 1994;7:1-8.

7 Eagger S, Luxon LM, Davies RA, et al. Psychiatric morbidity in patients with peripheral vestibular disorder: a clinical and neuro-otological study. F Neurol Neurosurg Psychiatry 1992;55:383-7.

8 Kroenke K, Lucas CA, Rosenberg ML, et al. Causes of persistent dizziness: a prospective study of 100 patients in sistent dizziness: a prospective study of 100 patient

9 Kroenke K, Lucas CA, Rosenberg ML, et al. Psychiatric Kroenke $\mathrm{K}$, Lucas CA, Rosenberg ML, et al. Psychiatric disorders and functional impairment in patients

10 Clark DB, Hirsch BE, Smith MG, et al. Panic in otolyngology patients presenting with dizziness or hearing loss. Am $\mathcal{F}$ Psychiatry 1994;151:1223-5.

11 Stein MB, Asmundson GJG, Ireland D, et al. Panic disorder in patients attending a clinic for vestibular disorders. $A m f$ Psychiatry 1994;151:697-700.

12 Frommberger VH, Tettenborn B, Buller R, et al. Panic disorder in patients with dizziness. Arch Intern Med 1994;154: $590-1$
13 Drachman DA, Hart CW. An approach to the dizzy patient. Neurology 1972;22:323-34.

14 Sloane PD. Dizziness in primary care: results from the National Ambulatory Medical Care Survey. F Fam Pract 1989;29:33-8.

15 Yardley L, Beech S, Zander L, et al. A randomised controlled trial of exercise therapy for dizziness and vertigo in primary care. Brf Gen Pract 1998;48:1136-40.

16 Sullivan M, Clark MR, Katon WJ, et al. Psychiatric and otoogic diagnoses in patients complaining of dizziness. Arch Intern Med 1993;153:1479-84.

17 Sloane PD, Hartman M, Mitchell CM. Psychological factors associated with chronic dizziness in patients aged 60 and older. 7 Am Geriatr Soc 1994;42:854-3.

18 Jacob RG, Lilienfeld SO, Furman JMR, et al. Panic disorder with vestibular dysfunction: further clinical observations and description of space and motion phobic stimuli. $\mathcal{F}$ Anxiety Disord 1989;3:117-30.

19 Jacob RG, Furman JM, Durrant JD, et al. Panic, agoraphobia and vestibular dysfunction. Am f Psychiatry 1996;153: 503-12.

20 Yardley L, Britton J, Lear S, et al. Relationship between balance system function and agoraphobic avoidance. Behav Res Ther 1995;33:435-9.

21 Yardley L, Luxon L, Bird J, et al. Vestibular and posturographic test results in people with symptoms of panic and agoraphobia. F Audiological Med 1994;3:48-65.

2 Sklare DA, Stein MB, Pikus AM, et al. Dysequilibrium and audiovestibular function in panic disorder: symptom profiles and test findings. Am f Otol 1990;11:338-41.

23 Jacob RG. Panic disorder and the vestibular system. Psychiatr Clin North Am 1988;11:361-74.

24 Jacob RG, Furman JM, Balaban CD. Psychiatric aspects of vestibular disorders. In: RW Baloh, GM Halmagyi, eds. Disorders of the vestibular system. Oxford;Oxford University Press, 1996:509-28.

25 Yardley L, Hallam R. Psychosocial aspects of balance disorders. In: Bronstein A, Brandt T, Woolacott M, eds. Clinical aspects of balance and gait disorders. London; Edward Arnold, 1996:251-67.

26 Clark MR, Sullivan MD, Fischl M, et al. Symptoms as a clue to otologic and psychiatric diagnosis in patients with dizziness. F Psychosom Res 1994;38:461-70.

27 Nashner LM. Computerized dynamic posturography. In: Jacobson GP, Newman CW, Kartush JM, eds. Handbook of balance function testing. St Louis: Mosby Year Book, 1993:280-307.

28 Lewis G, Pelosi AJ, Glover E, et al. The development of a computerized assessment for minor psychiatric disorder. computerized assessment for

29 Davis A. Hearing in adults. London: Whurr, 1995.

30 Working Group on Evaluation of Tests for Vestibular Function. Evaluation of tests for vestibular function. Aviat Space Environ Med 1992;63(suppl):A1-34.

31 Furman JM, Jacob RG. Psychiatric dizziness. Neurology 1997;48:1161-6.

32 Lewis G. Assessing psychiatric disorder with a human interviewer or a computer. 7 Epidemiol Community Health 1994; 48:207-10.

33 Yardley L, Luxon LM, Haacke NP. A longitudinal study of symptoms, anxiety, and subjective wellbeing in patients with vertigo. Clin Otolaryngol 1994;19:109-16.

34 Brandt Th. Phobic postural vertigo. Neurology 1996;46: 1515-19.

35 Yardley L, Higgins M. Spatial updating during rotation: the role of vestibular information and mental activity. $\mathcal{F}$ Vestib Res 1998;8:1-8.

36 Lajoie Y, Teasdale N, Bard C, et al. Attentional demands for static and dynamic equilibrium. Exp Brain Res 1993;97: $139-44$ 